\title{
The INO80 chromatin remodeling complex prevents polyploidy and maintains normal chromatin structure at centromeres
}

\author{
Anna L. Chambers, ${ }^{1,4}$ Georgina Ormerod, ${ }^{1,4}$ Samuel C. Durley, ${ }^{2}$ Tina L. Sing, ${ }^{3}$ Grant W. Brown, ${ }^{3}$ \\ Nicholas A. Kent, ${ }^{2}$ and Jessica A. Downs ${ }^{1,5}$ \\ ${ }^{1}$ MRC Genome Damage and Stability Centre, University of Sussex, Brighton BN1 9RQ, United Kingdom; ${ }^{2}$ Cardiff School \\ of Biosciences, Cardiff University, Cardiff CF10 3US, United Kingdom; ${ }^{3}$ Department of Biochemistry, Donnelly Centre for Cellular \\ and Biomolecular Research, University of Toronto, Toronto, Ontario M5S 3E1, Canada
}

The INO80 chromatin remodeling complex functions in transcriptional regulation, DNA repair, and replication. Here we uncover a novel role for INO80 in regulating chromosome segregation. First, we show that the conserved Ies6 subunit is critical for INO80 function in vivo. Strikingly, we found that loss of either Ies6 or the Ino80 catalytic subunit results in rapid increase in ploidy. One route to polyploidy is through chromosome missegregation due to aberrant centromere structure, and we found that loss of either Ies6 or Ino80 leads to defective chromosome segregation. Importantly, we show that chromatin structure flanking centromeres is altered in cells lacking these subunits and that these alterations occur not in the Cse4-containing centromeric nucleosome, but in pericentric chromatin. We provide evidence that these effects are mediated through misincorporation of H2A.Z, and these findings indicate that H2A.Z-containing pericentric chromatin, as in higher eukaryotes with regional centromeres, is important for centromere function in budding yeast. These data reveal an important additional mechanism by which INO80 maintains genome stability.

[Keywords: INO80; Ies6; polyploidy; centromere; chromosome segregation; H2A.Z]

Supplemental material is available for this article.

Received July 2, 2012; revised version accepted October 18, 2012.

Centromeres are specialized chromatin structures that provide the platform for kinetochore complex attachment during mitosis. The kinetochores in turn form attachments with the mitotic spindles, which are required for segregation of chromosomes. Appropriate chromosome segregation is critical to prevent chromosomal instability. Problems with centromere-kinetochore attachments lead to defects in chromosome segregation, and this can result in aneuploidy (Verdaasdonk and Bloom 2011). Chromosomal instability (and aneuploidy) is prevalent in cancer cells and correlates with poor prognosis (Foijer 2010). Understanding how centromere structure contributes to proper chromosome segregation therefore has important therapeutic implications.

Centromeres in many organisms, including mammals and fission yeast, are large and contain repetitive DNA sequences. They contain multiple nucleosomes with the

\footnotetext{
${ }^{4}$ These authors contributed equally to this work.

${ }^{5}$ Corresponding author

E-mail j.a.downs@sussex.ac.uk

Article is online at http://www.genesdev.org/cgi/doi/10.1101/gad.199976.112.
}

centromere-specific histone $\mathrm{H} 3$ variant CENP-A, which is required for kinetochore attachment, and these are termed "regional" centromeres (Torras-Llort et al. 2009; Verdaasdonk and Bloom 2011). The H2A.Z histone variant is enriched in the centromeres and pericentric heterochromatin of mammals and is an important component of the three-dimensional structure of the mouse centromere (Greaves et al. 2007). H2A.Z enrichment has also been found in the pericentric heterochromatin of fission yeast, suggesting that this may be a conserved constituent of regional centromeres (Kim et al. 2009). In both mammals and fission yeast, H2A.Z depletion leads to chromosome segregation defects (Carr et al. 1994; Rangasamy et al. 2003; Greaves et al. 2007; Hou et al. 2010), suggesting that H2A.Z contributes to proper centromere-kinetochore attachments during mitosis (Boyarchuk et al. 2011).

The small, single-nucleosome "point" centromeres of budding yeast contain a single CENP-A (Cse4) variantcontaining nucleosome and are not flanked by heterochromatin or large repetitive stretches of DNA, making them quite distinct from both mammalian and fission 
yeast regional centromeres (Albert et al. 2007; TorrasLlort et al. 2009; Verdaasdonk and Bloom 2011). Moreover, H2A.Z is not found in the centromeric nucleosome (Albert et al. 2007). However, a number of studies point to a role for H2A.Z in mediating faithful chromosome segregation in this organism as well. First, the gene encoding H2A.Z (HTZ1) was identified in screens for synthetic-lethal and synthetic dosage-lethal interactions with kinetochore mutants (Measday et al. 2005). In addition, H2A.Z was found enriched in chromatin flanking budding yeast centromeres, and loss of H2A.Z led to chromosome segregation defects (Krogan et al. 2004). More recently, the enrichment of H2A.Z flanking the centromeres was found to be impaired in mutants that have defective kinetochore attachment (Kawashima et al. 2011), raising the possibility that H2A.Z also contributes to the structure and function of point centromeres.

The INO80 chromatin remodeling complex facilitates transcriptional regulation, DNA repair, and replication (Bao and Shen 2007; Conaway and Conaway 2009). Recently, it was shown that INO80 has a histone exchange activity in vitro and is capable of replacing H2A.Z/H2B dimers with H2A/H2B dimers in nucleosomes (PapamichosChronakis et al. 2011). Consistent with a role in regulating H2A.Z localization in vivo, the genome-wide distribution of H2A.Z is altered in the absence of INO80 (PapamichosChronakis et al. 2011).

There are 15 subunits in the budding yeast INO80 complex, including the catalytic subunit, also called Ino80. There are two proteins with homology with prokaryotic RuvB (Rvb1 and Rvb2), actin (Act1), and three actin-related proteins (Arp4, Arp5, and Arp8). These proteins are all conserved in the mammalian INO80 complex, along with Ies2 and Ies6. In contrast, the Nhp10, Taf14, Ies1, Ies3, Ies4, and Ies5 subunits are not conserved in INO80 in higher eukaryotes. Relatively little is known about the function of many of these subunits.

We set out to investigate the contribution of the Ies1-6 subunits to INO80 activity. In doing so, we established that the Ies6 subunit is critical to INO80 function in vivo. Strikingly, we found that loss of either the Ies6 or Ino80 subunit of INO80 results in a rapid progression in the population to polyploidy and, consistent with a role in centromere function, also leads to chromosome segregation defects. Moreover, we found that the position of the centromeric nucleosome appears to be normal in the absence of Ies6 or Ino80, but that chromatin structure flanking centromeres is altered in ino80 and ies6 mutant cells. Notably, the effects of Ino80 and Ies6 on chromosome segregation and ploidy are far more dramatic than in yeast lacking H2A.Z altogether and led us to a model in which aberrant or increased localization of H2A.Z (rather than loss) leads to defective centromere function and rapid alterations in ploidy. In support of this model, we found that H2A.Z enrichment in pericentric chromatin is altered in ies 6 and ino80 mutants. Moreover, H2A.Z overexpression accelerates the shift, and reduced H2A.Z expression delays the shift to higher ploidy in ies6 mutant cells. Together, these data demonstrate that INO80 functions to promote the fidelity of chromosome segregation and thus uncover an additional mechanism by which INO80 prevents genome instability.

\section{Results}

\section{Ies6 is critical for INO80 function in vivo}

In order to investigate the relative contribution of an understudied subset of INO80 subunits to replicative stress, we tested the sensitivity of strains lacking the Ies1, Ies2, Ies3, Ies4, Ies5, and Ies6 subunits to hydroxyurea (HU). Most of these strains show no substantial difference in HU hypersensitivity compared with wild type (Fig. 1A). The two exceptions to this were the ies2 and ies6 mutant strains (Fig. 1A). Because the phenotype of the ies2 mutant strain was relatively mild, we decided to focus our studies on the ies6 mutant strain.

We compared the ies6 mutant strain directly with a strain lacking Ino80, the catalytic subunit of the complex, and found that the level of hypersensitivity is comparable (Fig. 1B). Previous studies found that strains lacking the Arp8 subunit of INO80 are phenotypically similar to ino80 mutant strains, and the purified INO80 complex from cells lacking Arp8 is compromised for catalytic activity in vitro (Shen et al. 2003). We also compared the ies6 mutant strain with an arp8 deletion and found that, like ino80, the ies6 mutant strain shows a more severe hypersensitivity to $\mathrm{HU}$ than the arp 8 mutant strain (Fig. 1B). These data demonstrate that the Ies6 subunit is critical for the function of the INO80 complex during periods of replicative stress in vivo.

\section{Rapid increase in ploidy upon loss of IES6 or INO80}

To further investigate the role of Ies6 in facilitating INO80 function during replicative stress, we set out to establish conditions for synchronous release into $S$ phase. In doing so, we made the unexpected discovery that our haploid ies6 deletion strain had a diploid profile when cells were analyzed by fluorescence-activated cell sorting (FACS) (Fig. 2A). We tested several independently derived

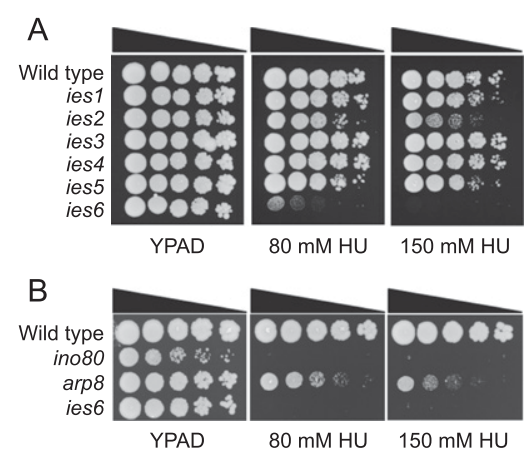

Figure 1. The ies6 mutant strain shows HU hypersensitivity similar to the ino80 mutant strain. $(A, B)$ Serial dilutions of mid$\log$ cultures of the indicated strains were plated onto medium containing the indicated amounts of HU. Plates were imaged after $5 \mathrm{~d}$ at $30^{\circ} \mathrm{C}$. 
A

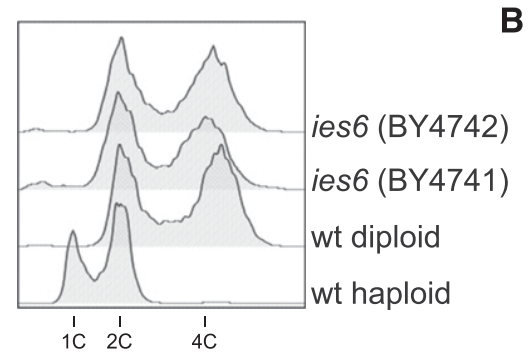

B

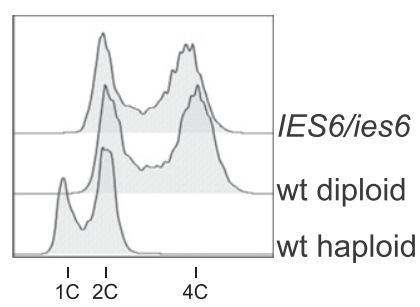

C
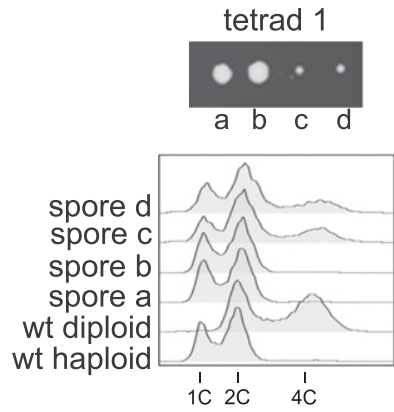

D

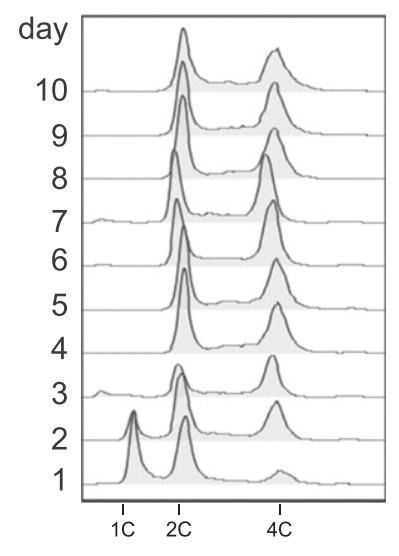

tetrad 2
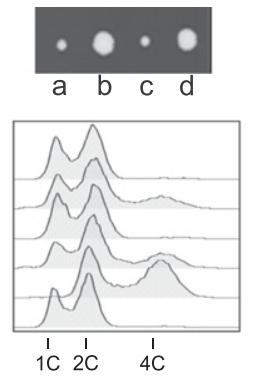

E
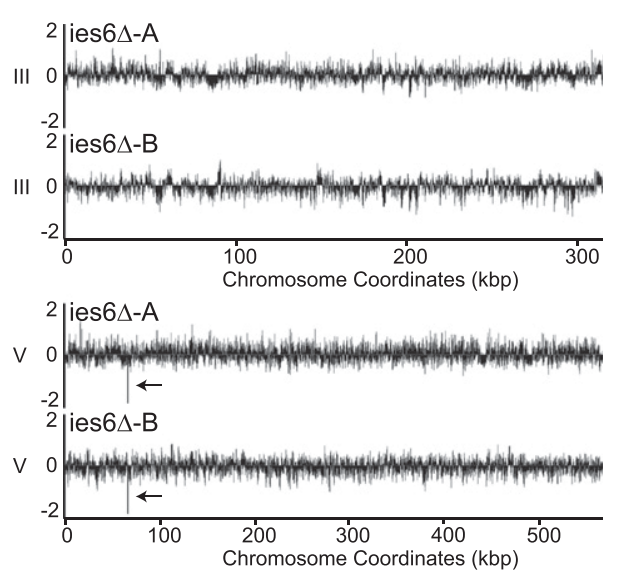

Figure 2. Loss of IES6 leads to rapid increase in ploidy. (A) FACS analysis of mid-log cultures of BY4741 (wild-type [wt] haploid), BY4743 (wildtype diploid), and ies6-null strains in the BY4741 or BY4742 backgrounds. (B) FACS analysis of the IES6/ies6 heterozygous diploid relative to haploid and diploid wild-type strains. (C, top panels) Tetrad dissection of spores from the IES6/ies6 heterozygous diploid showing 2:2 segregation of the ies6::KanR disruption. (Bottom panels) FACS analysis of mid-log cultures corresponding to the newly sporulated tetrads. (D) FACS analysis of an ies6-null strain, newly generated by sporulation, over time. (E) Comparative genomic hybridization of a newly generated ies6-null strain after 40 generations, which indicates a full genome duplication. The $\log 2$ ratio of signal of sample over wild-type control is displayed for the indicated chromosomes. The deletion of IES6 is indicated by arrows on chromosome $\mathrm{V}$ and acts as an internal control for the experiment. ies6 deletion strains either generated in our laboratory or obtained from other sources and found that all showed the same FACS profile (Fig. 2A; data not shown). We found that the cells that were created in a MATa parent strain still responded normally to $\alpha$ factor, unlike MATa/ $M A T \alpha$ diploid cells (data not shown). This suggested that the ies6 haploid strains had MATa/MATa gene expression and therefore had undergone a genome duplication.

We considered two possible explanations for the "diploid" status of the ies6-null strains. The first possibility was that ies6-null strains are only viable when the genome has undergone a duplication event. This was posited because of the slow growth and aberrant morphology observed with the ies6-null strains (see Figs. 1, 2C, 4 [below]), combined with the fact that we were unable to find a ies6-null strain with haploid DNA content in a variety of strain backgrounds. Alternatively, we considered that Ies6 may function to maintain normal ploidy, and in its absence, the cells rapidly become polyploid.
To discriminate between these possibilities, we sporulated a heterozygous ies6/IES6 diploid strain. First, we established that the heterozygous strain had a normal diploid profile when assayed by FACS (Fig. 2B). Upon sporulation and tetrad dissection, we found that there was no loss of viability among the spores, and the spores showed a 2:2 segregation of the ies6 deletion (Fig. 2C). The ies6-null cells grew very slowly compared with the wild-type haploids, similar to the ies6 strains that were generated by gene disruption. When we examined these newly generated ies6 haploid cells by FACS, we found that a substantial subpopulation of cells in these ies6-null strains still show a haploid profile (Fig. 2C). This result demonstrates that cells lacking IES6 can exist as haploids and rules out the first possibility: that genome duplication is required for viability in the absence of IES6.

To explore the second possibility-that loss of IES6 removes a barrier to polyploidy-newly sporulated ies6null cells were analyzed by FACS over time. We again saw that many of the cells in the population are haploid at 
early time points and found that there is a rapid increase in ploidy over time (Fig. 2D). We determined that the population becomes fully diploid within $\sim 30$ generations, consistent with the possibility that Ies6 functions to maintain normal ploidy. While the FACS analysis is consistent with a full genome duplication, it is insufficiently sensitive to determine whether there are more subtle genome alterations. We therefore performed comparative genomic hybridization on tiling microarrays to analyze the ies6-null genome at higher resolution. This was done at 40 generations, at which point this population is fully diploid (Fig. 2E), and again at 80 generations post-sporulation (Supplemental Fig. $\mathrm{S} 1 \mathrm{~A})$, and the results demonstrate that the cells have indeed undergone a full genome duplication and are diploids.

To determine whether this effect on ploidy is unique to Ies6 or common to all subunits of the INO80 complex, we examined our other INO80 mutant strains by FACS. The ies1, ies2, ies3, ies4, and ies5 mutant strains all showed a normal haploid profile (Fig. 3A). In contrast, we found that the haploid ino80-null strain had a diploid FACS profile, similar to the ies6-null (Fig. 3B), suggesting that the effect of IES6 loss on ploidy is likely to be due to its role as a part of the INO80 chromatin remodeling complex. We also tested the arp8-null strain, but surprisingly, given the importance of Arp8 to INO80 function, found that the $\operatorname{arp} 8$ strain did not display a diploid FACS profile (Fig. 3B). This suggests that the role of INO80 in preventing polyploidy is distinct from its role in responding to replicative stress, which is Arp8-dependent
(Papamichos-Chronakis and Peterson 2008; Shimada et al. 2008; Falbo et al. 2009; Papamichos-Chronakis et al. 2011).

To further investigate the contribution of the Ino80 subunit to maintaining normal ploidy, we sporulated a heterozygous INO80/ino80 diploid and followed the newly generated ino80-null spores by FACS over time as above. We obtained a profile similar to the ies 6 cells (Fig. 3C). Moreover, the ino80-null strain was analyzed using comparative genomic hybridization on tiling microarrays as above. In this case, we chose to analyze the spores at 20 and 40 generations post-sporulation to reflect the faster appearance of diploids in the population and found that, like the ies6 mutants, the ino80-null strains have also undergone a full genome duplication (Fig. 3D; Supplemental Fig. S2A). Notably, we also found that there was a heterozygous Hawthorne deletion (Hawthorne 1963), resulting from recombination between the MAT $\alpha$ and HMRa loci on the right arm of chromosome III, in one of the two spores analyzed (Fig. 3D; Supplemental Fig. S2C). INO80 is enriched at the MAT locus and regulates transcription of $M A T \alpha 1$ and $M A T \alpha 2$ (Tsukuda et al. 2005). It is possible that in its absence, the region is more amenable to promiscuous recombination due to altered chromatin structure, but this will require further investigation. Together, these data demonstrate that both the Ino80 and Ies6 subunits of INO80 function to prevent increases in ploidy in cells.

DNA damage sensitivity and decreased fitness are associated with increases in ploidy (Andalis et al. 2004; Storchova et al. 2006). We therefore considered the
A

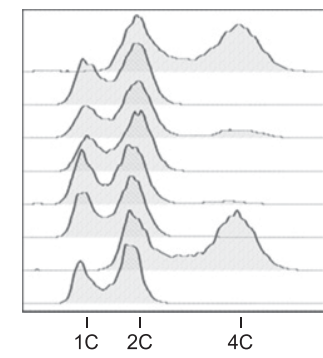

C

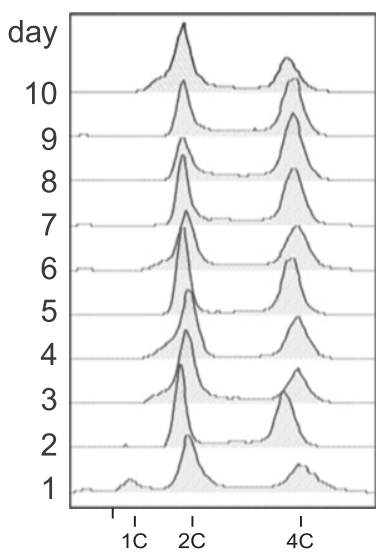

B

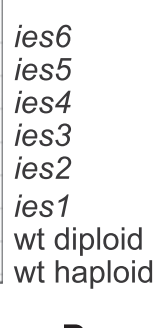

D
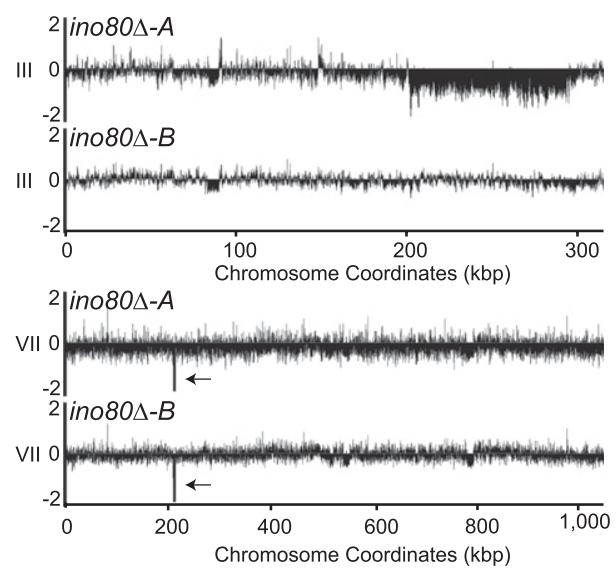

Figure 3. Loss of $I N O 80$, but not $A R P 8$ or IES1-5, also results in increased ploidy. $(A, B)$ FACS analysis of mid-log cultures of the indicated haploid strains. (C) FACS analysis of an ino80-null strain, newly generated by sporulation, over time. $(D)$ Comparative genomic hybridization of a newly generated ino80-null strain after 20 generations. The $\log 2$ ratio of signal of sample over wild-type control is displayed for the indicated chromosomes. The deletion of INO80 is indicated by arrows on chromosome VII and acts as an internal control for the experiment. 
possibility that some or all of the phenotypes observed in the ies6 and ino80 mutant strains may be attributable to the increase in ploidy. We therefore reintroduced the wild-type IES6 gene into the ies6-null strain and compared this with a wild-type haploid strain. We found that, as expected, reintroduction of IES6 had no effect on ploidy, and the cells remained stably diploid over time (Fig. 4A). Importantly, we found that the HU hypersensitivity of the ies6-null strain was restored to apparently wild-type levels when the IES6 gene was present (Fig. 4B), despite the fact that these cells still had higher ploidy than the haploid wild-type control. We also observed that reintroduction of the IES6 gene restored normal growth rate and morphology (Fig. 4B,C; data not shown). These data suggest that the phenotypes observed in the ies6 mutant cells are not an indirect effect of increased ploidy but instead result from loss of INO80 chromatin remodeling activity.

The ies6 and ino80 mutant strains appear to remain stably diploid over time, and we did not see the appearance of tetraploid (or higher ploidy) cells in the population. One possible explanation for this effect is that the strains are undergoing inappropriate recombination or transcriptional regulation of the MAT locus, resulting in the ability of the mutant populations to self-mate. This would result in an apparent genome duplication and also account for the lack of higher levels of ploidy. Strains that have self-mated should no longer be competent to mate,
A

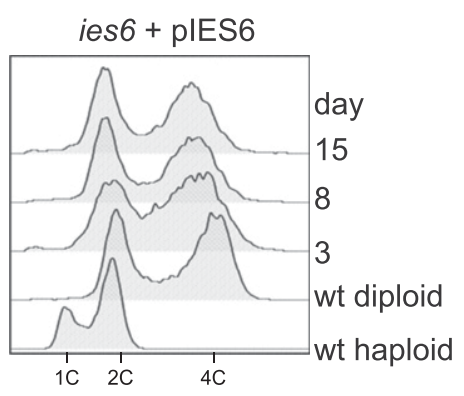

B

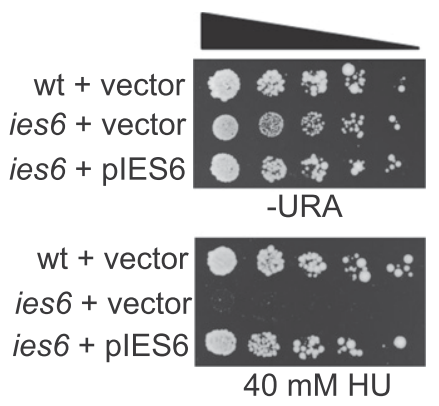

C

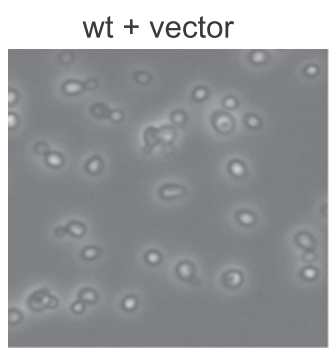

D

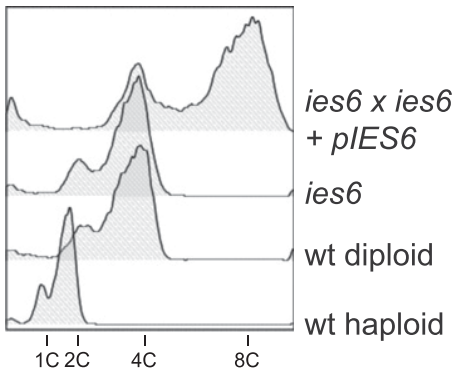

ies6 + vector

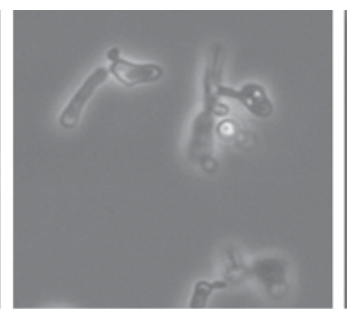

E

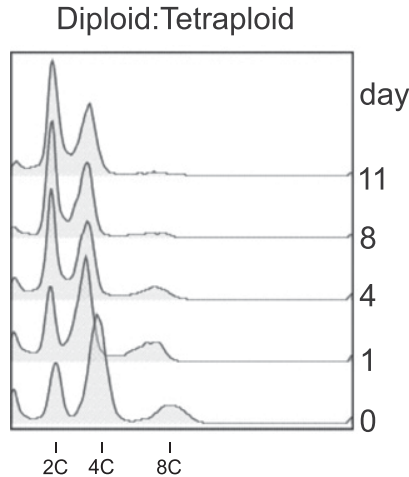

ies $6+\mathrm{plES} 6$

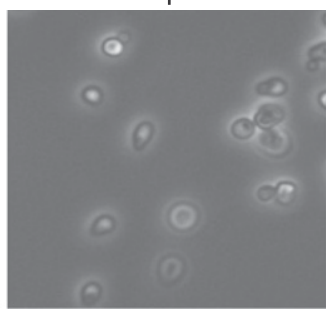

$\mathbf{F}$

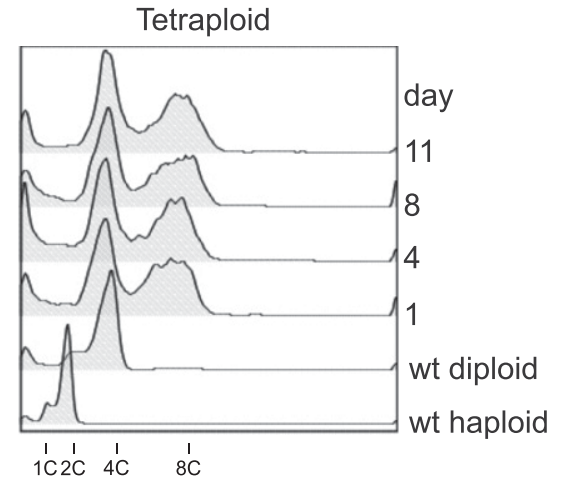

Figure 4. Altered ploidy does not affect HU sensitivity or morphology but does affect fitness. (A) FACS analysis of ies6-null strain complemented with wild-type (wt) IES6 (pJD640; pIES6) over time. (B) Serial dilutions mid-log cultures of haploid wild type + empty vector, ies6 + empty vector, and ies6 + IES6 (pJD640; pIES6) plated onto medium containing the indicated amount of HU. Plates were imaged after $5 \mathrm{~d}$ at $30^{\circ} \mathrm{C}$. $(C)$ Phase-contrast microscopy images of haploid wild type + empty vector, ies $6+$ empty vector, and ies $6+$ wild-type IES6 (pJD640; pIES6). (D) Creation of an ies6-null tetraploid strain by mating two diploid ies6-null strains, analyzed by FACS. (E) Equal numbers of ies6 diploid and ies6 tetraploid cells were mixed (day 0) and grown with continuous subculturing for $11 \mathrm{~d}$. Samples were analyzed by FACS at the indicated time points. $(F)$ Tetraploid ies6-null cells were grown with continuous subculturing, and samples were analyzed by FACS at the indicated time points. 
so we therefore tested fully diploid colonies and found that both ino80 and ies6 diploid strains were able to mate with one of the two mating type strains they were tested against (Supplemental Fig. S3A), suggesting that this is not the mechanism by which the strain populations become diploid.

In order to gain further insight into why the population apparently stabilizes after a single full genome duplication, we investigated whether the ies6-null cells were able to exist as tetraploids. We took two "diploid" strains of opposite mating type, where one strain had a copy of the wild-type IES6 gene on a URA3-containing plasmid, and mated them. The resulting strains were tetraploid when analyzed by FACS (Fig. 4D). The strains were able to grow when restreaked onto 5-FOA to select for loss of the wild-type IES6-containing plasmid, demonstrating that Ies6 is not required for viability in a tetraploid cell (data not shown).

As described above, higher ploidy is associated with decreased fitness. To test whether this accounted for the lack of tetraploids in the ies6 population, we mixed equal numbers of ies6-null "diploid" and tetraploid cells and kept them in mid-log phase by repeated subculturing. We found that the tetraploid population disappeared from the culture over time (Fig. 4E). This was not a consequence of unstable ploidy in the tetraploid cells, since a tetraploid population analyzed in parallel showed no change in FACS profile over time (Fig. 4F). Rather, these data suggest that tetraploid (and potentially higher-ploidy) cells may arise in the population but have decreased fitness and are effectively outcompeted by the diploid cells. In support of this, we note that the tetraploid strain consistently has a noticeable sub-G1 population in the FACS profile (Fig. 4F), which probably reflects a high percentage of inviable cells.

\section{Ies6 and Ino80 function to promote proper chromosome segregation}

A previous study identified a specific class of histone $\mathrm{H} 2 \mathrm{~A}$ mutants, including hta1-200 (S20F) and hta1-300 (G30D), that displayed an "increase in ploidy" phenotype to a stable diploid content in the population similar to that seen with ies6 and ino80 mutants (Pinto and Winston 2000). The H2A mutants had chromosome segregation defects caused by defective centromeric chromatin, suggesting a mechanism for the increase in ploidy. Interestingly, the "increase in ploidy" phenotype could be partly suppressed by overexpression of Arp4, a subunit of INO80 (Pinto and Winston 2000). In addition, a recent study found a patch on histone $\mathrm{H} 4$ that, when mutated, results in strains with chromosome segregation defects and "increase in ploidy" phenotypes, and these strains were also shown to have defects in centromeric chromatin structure (Yu et al. 2011). Finally, IES6 was identified in a screen for genes required for chromosome segregation (Measday et al. 2005), and INO80 shows a genetic interaction with the MAD1 spindle assembly checkpoint gene (Collins et al. 2007). We therefore set out to determine whether our ies6 and ino80 mutant cells might also have defects in chromosome segregation and centromeric chromatin structure.

We first tested the ability of the mutant strains to survive in the presence of the microtubule-destabilizing drug benomyl and found that, similar to strains with defective centromere or kinetochore structure (Kawashima et al. 2011; Storchova et al. 2011), both ies6 and ino80 strains are hypersensitive compared with wild type (Fig. 5A). We also stained asynchronously growing cells with DAPI to examine the distribution of nucleic acids in these strains. Both the ino80 and ies6 mutant cells show abnormal staining patterns compared with wild type (Fig. 5B), which could be indicative of chromosome segregation defects. In contrast, the arp 8 mutant strain had normal morphology and DAPI staining patterns (Fig. 5B).

To look more specifically at chromosome segregation, we investigated the ability of the ies6 and ino80-null cells to maintain a centromere-containing plasmid in the absence of selection. At the same time, we investigated the ability of these strains to maintain a $2-\mu \mathrm{m}$ plasmid, which does not rely on centromeric sequences for segregation but has a plasmid-specific partitioning mechanism. We found a clear decrease in the retention of the CEN-containing plasmid in both mutant strains relative to wild type (Fig. 5C). The $2-\mu \mathrm{m}$ plasmid is not retained as efficiently as the CEN plasmid in the wild-type strain. Nevertheless, there is no further reduction in this efficiency in either the ies6 or ino80 mutant strains (Fig. 5C), which suggests that the defect in retention is specific to centromere-containing plasmids. Together, these data suggest that the ino 80 and ies 6 mutant strains have a chromosome segregation defect.

While loss or gain of individual chromosomes would not be detectable by FACS, these results appear to be somewhat at odds with the comparative genomic hybridization (CGH) data, which suggest that the ies6 mutant population derived from a haploid cell is entirely diploid. One possibility is that there is frequent loss of individual chromosomes in the population, but that these aneuploid cells-in the absence of any substantial selective advantage-will be either sufficiently heterogeneous that they are essentially undetectable by CGH or, in many cases, inviable. To investigate this possibility, we looked at cell viability in mid-log cultures of wild-type and ies6-null strains using either Trypan blue staining or colony counting. Using both methods, we found that a substantial proportion of the ies 6 mutant cells in mid-log cultures is not viable (Table 1; data not shown). This result is consistent with frequent lethality caused by chromosome missegregation in the population.

\section{Ies6 and Ino80 function to maintain chromatin structure at centromeres}

There are multiple cellular pathways that impact on chromosome segregation. For example, chromosome missegregation can be caused by defects in microtubule assembly or spindle formation. We therefore investigated whether this might be the mechanism by which the 
A
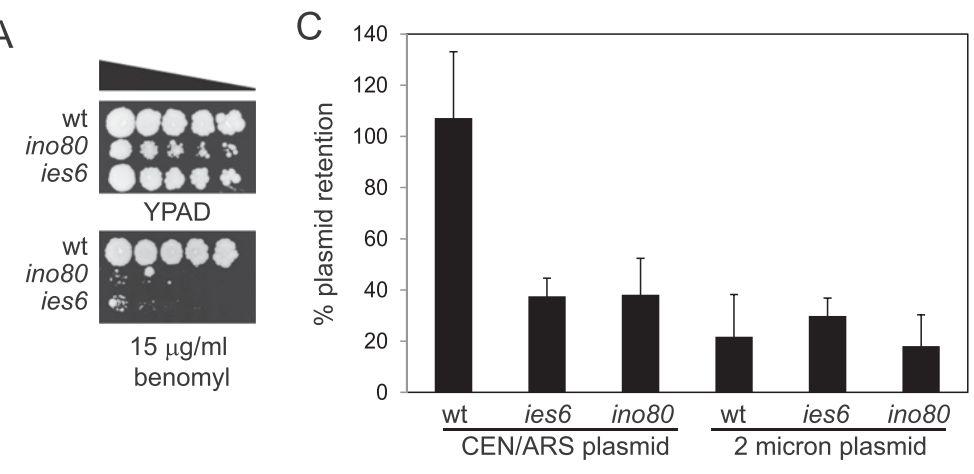

B
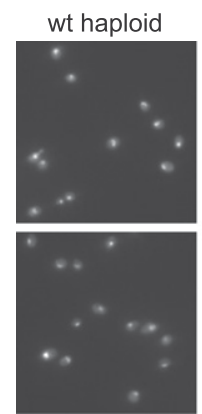
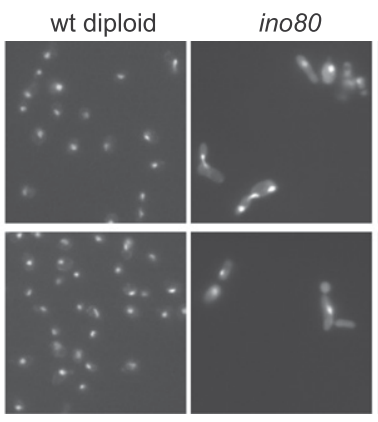

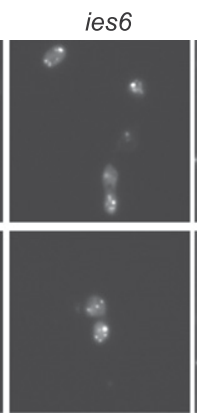

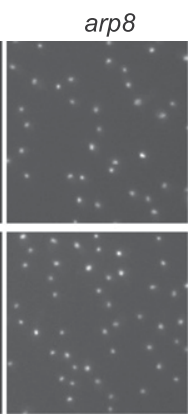

Figure 5. Ino 80 and Ies 6 are important for normal chromosome segregation. (A) Serial dilutions of midlog cultures of wild-type (wt) (BY4741) or ino80 or ies6 mutant strains on medium containing 0 or $15 \mu \mathrm{g} / \mathrm{mL}$ benomyl. Plates were imaged after $5 \mathrm{~d}$ at $30^{\circ} \mathrm{C} .(B)$ DAPI-stained haploid wild-type (BY4741), diploid wild-type (BY4743), or ino80-, ies6-, or arp8-null cells. (C) Retention of a CEN/ARS or $2-\mu \mathrm{m}$ plasmid in wild-type (BY4741) and ino80- and ies6-null strains after $22 \mathrm{~h}$ growth in the absence of selection. Data shown are the mean survival of a minimum of three colonies per strain assayed on selective medium relative to nonselective medium $\pm 1 \mathrm{SD}$. ino80 or ies6 mutant strains become polyploid. To do this, we performed immunofluorescence using antitubulin to analyze the spindle morphology (Supplemental Fig. S3B). When compared with wild-type cells, we could not detect any gross alterations in spindle assembly or morphology in ino80 mutants. We also looked at spindle pole body (SPB) duplication using a strain with GFP-tagged Spc42. We found that disruption of IES6 in this background did not detectably change SPB duplication (Supplemental Fig. S3C). While we cannot absolutely rule out an effect of spindle assembly in these strains, this does not appear to be the driving force behind the chromosome segregation defects and increase in ploidy phenotype of strains lacking INO80 or IES6.

Another route to chromosome segregation defects and altered ploidy is through defects in centromere structure. INO80 can remove H2A.Z/H2B dimers from nucleosomes and replace them with $\mathrm{H} 2 \mathrm{~A} / \mathrm{H} 2 \mathrm{~B}$ dimers (PapamichosChronakis et al. 2011). As previously described, H2AZ is found enriched in chromatin flanking centromeres (Supplemental Fig. S4A; data from Albert et al. 2007), and H2A.Z enrichment in chromatin is altered in an ino80 mutant strain (Papamichos-Chronakis et al. 2011). In addition, Ino80 was identified in a purification of the centromeric Cse4 protein (Ranjitkar et al. 2010).

One attractive explanation, therefore, for the segregation defects in the absence of Ino80 and Ies6 is altered chromatin structure at centromeres in the mutant strains. In order to directly investigate this possibility, chromatin from wild-type and ies6 and ino80 mutant strains was isolated and analyzed at two different centromeres by indirect end labeling to map the positions of the nucleosomes. We found that the location and pattern of pro- tection of the Cse4-containing (CENP-A) centromeric nucleosome was not visibly altered in the ies6 or ino80 mutant strains relative to wild-type (Fig. 6A,B). However, there were clear differences in the position of MNasehypersensitive sites flanking each centromere. An additional hypersensitive site is visible on the NTH1-proximal (right) side of CEN4 in both ino80 and ies6 mutant strains (Fig. 6A). At CEN12, several subtle changes in signal intensity of existing sites and the appearance of a new hypersensitive site are apparent in both mutant strains on the YLR001C-proximal (right) side of the centromere (Fig. 6B).

We performed chromatin immunoprecipitation (ChIP) assays to investigate whether H2A.Z enrichment at CEN4 or CEN12 was altered in the ino80 and ies6 mutant strains. First, we used an htz1-null strain to establish ChIP conditions and found that H2A.Z is incorporated into the chromatin flanking both CEN4 and CEN12 (Supplemental Fig. 4B). Using these conditions, we tested the ino80 and ies6 mutant strains and found that there is a substantial increase in enrichment of H2A.Z at these locations relative to the wild-type strain (Fig. 6C). This suggests that increased H2A.Z occupancy at centromeric

Table 1. Cell viability in mid-log cultures measured by Trypan blue staining

\begin{tabular}{lcc}
\hline Strain & $\begin{array}{c}\text { Nonviable } \\
\text { cells/total cells }\end{array}$ & Nonviable \\
\hline Wild-type haploid (BY4741) & $2 / 976$ & $0.2 \%$ \\
Wild-type diploid (BY4743) & $1 / 981$ & $0.1 \%$ \\
ies6 & $83 / 992$ & $8.4 \%$ \\
\hline
\end{tabular}



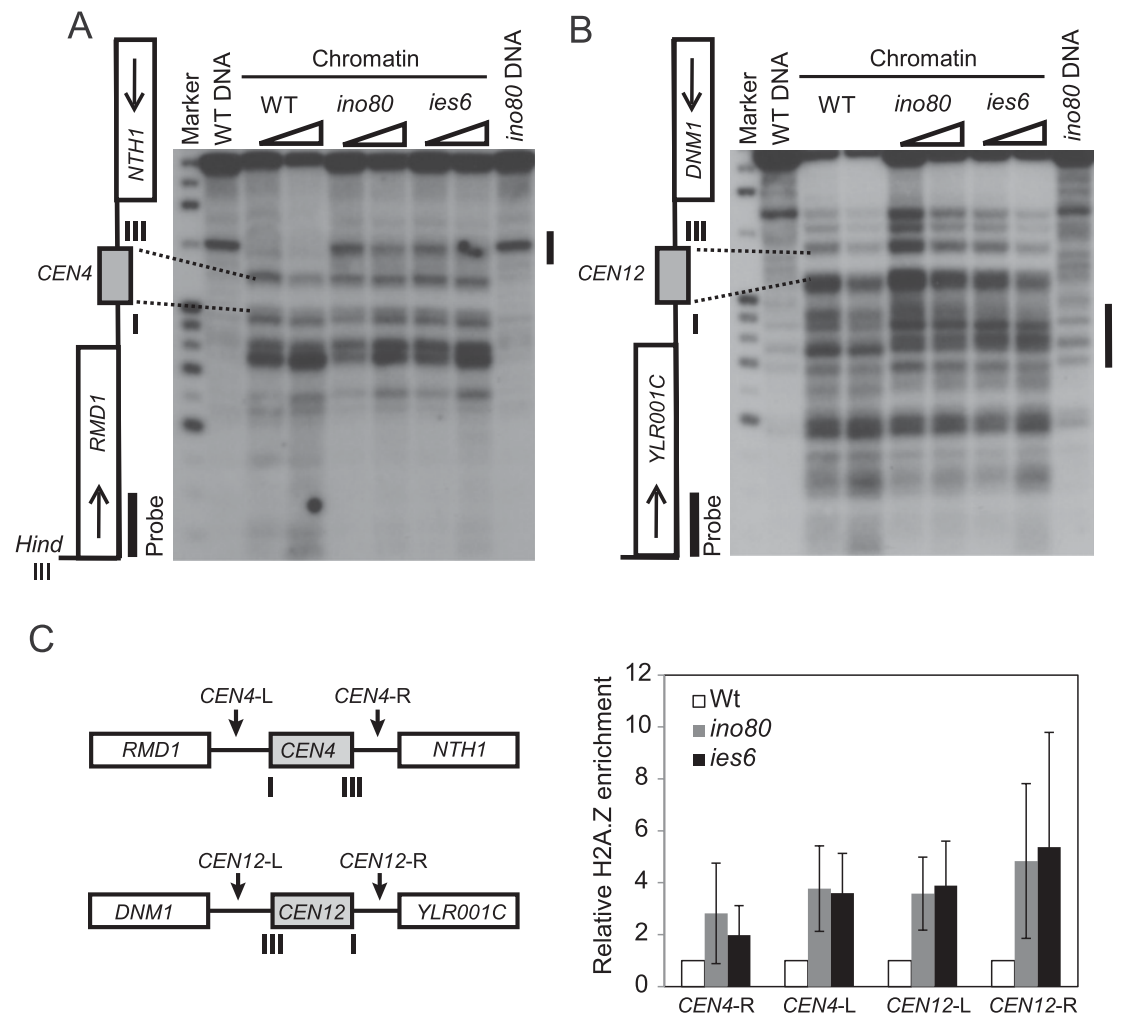

Figure 6. Ino80 and Ies6 are important for establishment of chromatin structure at pericentric regions. $(A)$ Indirect end label analysis of MNase accessibility at CEN4 in isogenic wild-type (wt) and ino80- and ies6null strains. Chromatin samples from each strain were digested with MNase at a concentration of 75 and $150 \mathrm{U} / \mathrm{mL}$. Deproteinized DNA controls from wild-type and ino80 strains are marked "DNA." The genomic restriction enzyme cleavage site and probe are marked on the locus map to the left of the blot. Dotted lines mark the MNaseresistant region corresponding to the CEN core particle. The region of Ino80/Ies6dependent MNase accessibility is marked to the right of the blot with a line. $(B)$ Indirect end label analysis of MNase accessibility at CEN12 in isogenic wild-type and ino80- and ies6-null strains as described for the previous panel. (C) ChIP of H2A.Z at CEN4 and CEN12 in isogenic wild-type and ino80- and ies6-null strains. The left panel shows schematics of CEN4 and CEN12 with arrows indicating the location of the primer pairs used for ChIP. The orientation of the CEN elements is also shown. The right panel shows the enrichment of H2A.Z at CEN4 and CEN12 in ino80-null (gray) and ies6-null (black) strains relative to the wild-type strain (white). ChIP signal was normalized to amplification of input DNA prior to calculation of relative enrichment. Data shown are the mean of at least three independent experiments $\pm 1 \mathrm{SD}$. chromatin may result in changes to the chromatin structure that are deleterious for correct segregation of chromosomes.

To test this possibility, we used an HTZ1 overexpression construct. Overexpression of H2A.Z should drive incorporation and/or mislocalization in the genome, particularly in the absence of Ino80 or Ies6 but also to some extent in a wild-type background. If INO80 is preventing changes in ploidy by removing inappropriate H2A.Z incorporation at centromeres, then H2A.Z overexpression should exacerbate the increase in ploidy phenotype of ino80 or ies6 mutant strains. To test this, we transformed a IES6/ies6 heterozygous diploid with a galactose-inducible $H T Z 1$ overexpression construct (or empty vector) and sporulated directly onto selective medium containing galactose to induce HTZ1 overexpression. The wild-type and ies 6 mutant spores were then grown for $10 \mathrm{~d}$ with continual subculturing followed by FACS. Notably, and consistent with a previous report (Sopko et al. 2006), overexpression of H2A.Z in wild-type cells results in the appearance of a small population of polyploid cells (Fig. 7A). Notably, the effect of H2A.Z overexpression on ies6-null cells was even greater. The ies6 mutant strain population carrying empty vector became fully diploid by day 7 under these growth conditions, whereas the population of ies6 mutant cells overexpressing H2A.Z was already a mix of haploid and diploid cells by day 1 and fully diploid by day 3 (Fig. 7B). This result supports the model in which misincorpora- tion of H2A.Z leads to chromosome missegregation and altered ploidy.

While deletion of HTZ1 can lead to segregation defects, htz1-null strains do not show a rapid increase in ploidy. This suggests that perhaps mislocalization or increased incorporation of H2A.Z at centromeres is more deleterious than its absence. If so, deletion of H2A.Z may rescue the ploidy defects found in the ino80 or ies 6 mutant strains. However, deletion of $h t z 1$ is synthetic-lethal with ino80 (Papamichos-Chronakis et al. 2011), and we were unable to obtain ies6/htz1 double mutants when a IES6/ ies6 HTZ1/htz1 strain was sporulated, suggesting htz1 deletion is also synthetic-lethal with ies6. However, by reducing HTZ1 expression by using a construct with a truncated promoter $\left(H T Z 1^{C P}\right)$, ino80 replication defects could be overcome (Papamichos-Chronakis et al. 2011). Because of marker differences, we were unable to use the same construct in our strain background but instead created a strain where the endogenous HTZ1 promoter was partly removed to leave the same 500-base-pair (bp) truncated promoter $\left(H T Z 1^{C P}\right)$ (Supplemental Fig. S4C). This did not result in the same degree of reduced H2A.Z expression as in Papamichos-Chronakis et al. (2011), but there was detectably lower expression relative to the wild-type control (Fig. 7C). This difference in expression levels is presumably due to the influence of the different genomic loci. The $H T Z 1^{C P}$ construct was introduced into our IES6/ies6 heterozygous diploid strain and sporulated. All four spores were followed over time by FACS post- 
A

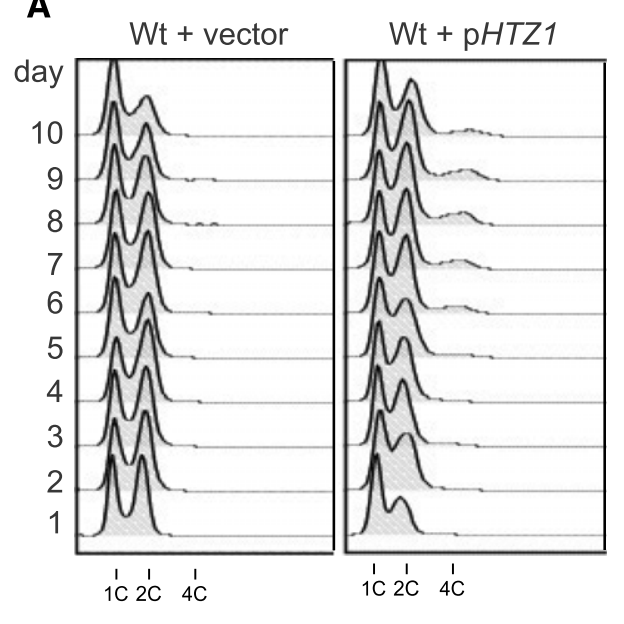

C

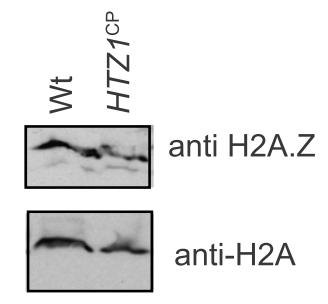

D

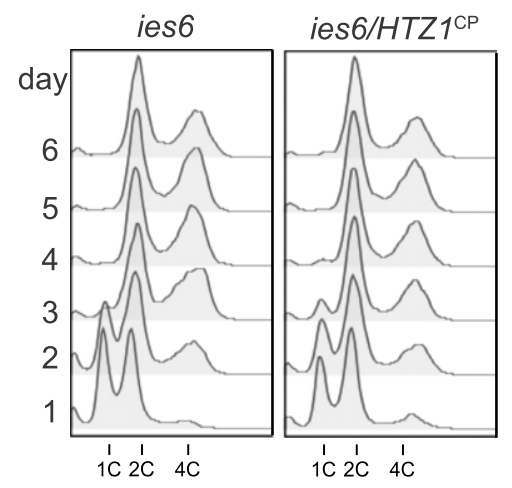

B

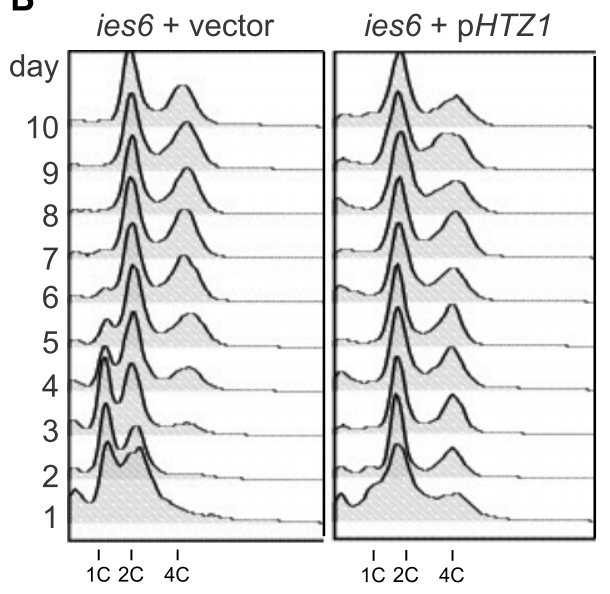

E

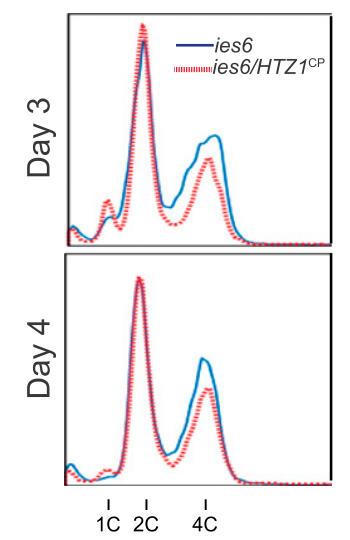

Figure 7. The "increase in ploidy" phenotype of the ies6-null strain is exacerbated by overexpression of H2A.Z and delayed by reduced expression of H2A.Z. $(A, B)$ Diploid strains containing a galactose-inducible HTZ1 overexpression construct (or empty vector) were sporulated and dissected onto selective medium containing galactose. These newly derived spores were continuously subcultured in selective medium containing galactose and analyzed over time by FACS. $(C)$ Western blot analysis of expression of $\mathrm{H} 2 \mathrm{~A} . \mathrm{Z}$ in wild-type (wt) and $H T Z 1^{C P}$ strains. Westerns were analyzed with anti-H2A.Z (top panel) and anti-H2A (bottom panel) as a loading control. (D) FACS analysis at $1,2,3,4,5$, and $6 \mathrm{~d}$ post-sporulation of wild-type, ies6, $H T Z 1^{C P}$, and ies6/HTZ1 ${ }^{C P}$ strains derived from a single tetrad. $(E)$ Overlays of the FACS data shown in $D$ for day 3 (top panel) and day 4 (bottom panel). The solid blue line shows the FACS profile of the ies6-null strain, and the dotted red line shows the FACS profile of the ies6/HTZ1 ${ }^{C P}$ strain.

sporulation, and there was no change in ploidy in the $H T Z 1^{C P}$ strain over the time course of the experiment (data not shown). Both the ies6 and ies6/HTZ1 ${ }^{C P}$ strains were fully diploid by day 5 (Fig. 7D), but the ies6/HTZ1 ${ }^{C P}$ strain had a delay in the progression, with a small but noticeable increase in the $1 \mathrm{C}$ content and reduction in $4 \mathrm{C}$ content on days 3 and 4 (Fig. 7D,E).

In addition to mislocalization of H2A.Z, global levels of H2A.Z acetylation are also substantially reduced in an ino80 mutant (Papamichos-Chronakis et al. 2011). Deletion of the gene encoding the HDAC subunit Hdal in either wild-type or ino80 strains results in hyperacetylated H2A.Z and also partly suppresses the HU sensitivity of an arp 8 mutant strain despite the fact that H2A.Z is still mislocalized (Papamichos-Chronakis et al. 2011). We therefore wanted to investigate whether deletion of $H D A 1$ would also rescue the changes in ploidy seen in the ino80 or ies6 mutant strains. The ino80/hda1 strain was reported to be extremely sick (Papamichos-
Chronakis et al. 2011), so we created a HDA1/hda1, IES6/ies6 heterozygous diploid strain and sporulated it in order to determine the effect of HDA1 deletion on ploidy in the absence of IES6. Four spores from a single tetrad (wild type, hda1, ies6, and ies6/hda1) were generated, and the levels of H2A.Z acetylated at K14 were measured. We found that, similar to the ino80 mutant strain, the ies6 mutant strain had substantially reduced acetylation of H2A.Z, and deletion of HDA1 did partly suppress this effect (Fig. 8A). However, deletion of HDA1 did not suppress the aberrant DAPI staining pattern of the ies6 mutant strain (Fig. 8B). Moreover, loss of hda1 does not rescue the benomyl sensitivity of the ies6 mutant strain (Fig. 8C). Interestingly, however, deletion of hda1 was able to weakly suppress the HU sensitivity of the ies6 mutant strain (Fig. 8C), which is consistent with the effect of hda1 deletion in an arp 8 strain on HU sensitivity (PapamichosChronakis et al. 2011). 
A

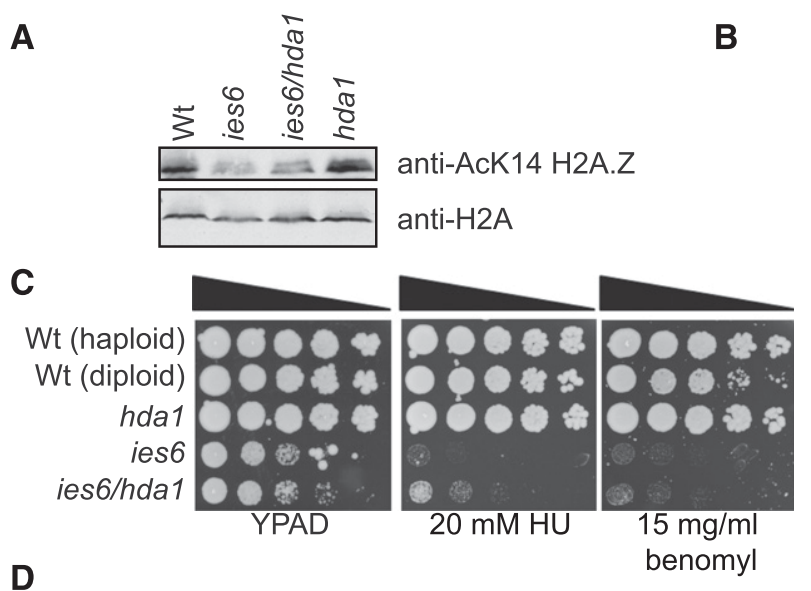

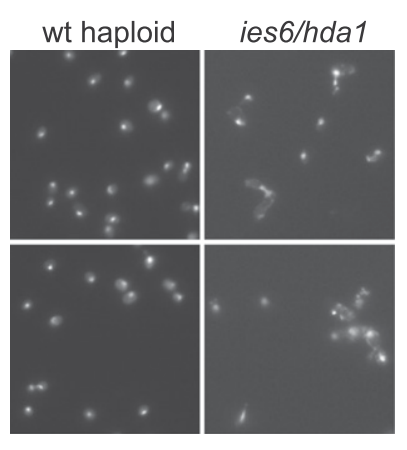

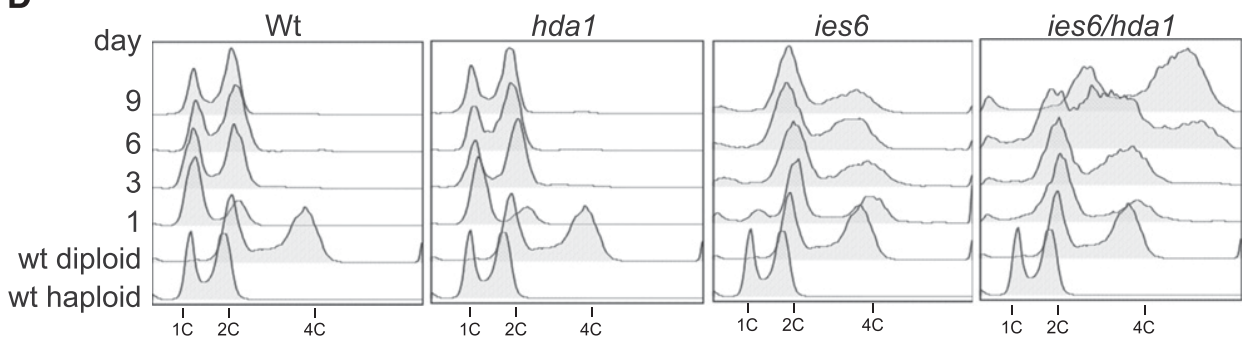

Figure 8. The "increase in ploidy" phenotype of the ies6-null strain is exacerbated by loss of Hda1. (A) Western blot analyses of acetylated K14 H2A.Z in wild-type (wt), ies6, ies6/hda1, hda1, and ino80 strains. Westerns were analyzed with anti-acetyl K14 H2A.Z (top panel) and anit-H2A (bottom panel) as a loading control. (B) DAPI-stained haploid wild-type (BY4741) or hda1/ies6 cells. (C) Serial dilutions of mid-log cultures of haploid wild-type (BY4741), diploid wild-type (BY4743), and hda1, ies6, and hda1/ies6 mutant strains on medium containing the indicated amount of HU or benomyl. (D) FACS analysis at 1, 3, 6, and 9 d post-sporulation of wild-type, hda1, ies6, and hda1/ies6 strains derived from a single tetrad.

We then analyzed the newly derived spores by FACS over time as above. First, we found that loss of HDA1 alone had no apparent effect on ploidy (Fig. 8D). Moreover, we found that the hda1/ies6 double mutant rapidly became diploid, similar to the ies6 single mutant (Fig. 8D), suggesting that $h d a 1$ deletion does not rescue the defect in maintaining ploidy of the ies6 mutant. Strikingly, we found that the hda1/ies6 showed further increases in ploidy at later time points (Fig. 8D), which may suggest that loss of HDA1 somehow overcomes the decreased fitness associated with tetraploidy in the ies6 mutant background. Together, these data suggest that, unlike replication defects, the chromosomal segregation defects associated with loss of ies 6 cannot be rescued by deletion of $H D A 1$, providing further evidence that the ability of INO80 to prevent chromosome missegregation is distinct from its role in replicative stress.

\section{Discussion}

We identified Ies6 as a critical subunit of the INO80 chromatin remodeling complex. Unlike other subunits of INO80, loss of IES6 phenocopies the ino80-null strain, suggesting that it is required for INO80 activity in vivo. INO80 is evolutionarily conserved, and a homologous complex has been isolated and characterized from human cells (Chen et al. 2011). The Ies6 subunit is conserved, and human Ies6 was found to be part of a subcomplex that assembles onto the ATPase domain of human Ino80 along with the Rvb homologs (Tip49a and Tip49b), hIes2, and hArp5 (Chen et al. 2011). Notably, however, Ies6 does not appear to be present in high-salt preparations of yeast INO80 that have chromatin remodeling activity in vitro (Shen et al. 2000, 2003). Given the severity of ies6 phenotypes, the lack of requirement for Ies 6 in remodeling assays in vitro is somewhat surprising, and we are currently investigating how this subunit plays such a critical role in vivo.

As described above, H2A.Z contributes to proper chromosome segregation in budding yeast (Krogan et al. 2004; Measday et al. 2005; Sopko et al. 2006; Kawashima et al. 2011), raising the possibility that, as in higher eukaryotes, H2A.Z contributes to the conformation of chromatin structure of centromeric regions. Our data show that the pericentric regions of budding yeast chromosomes 4 and 12 have aberrant chromatin structure when Ino80 or Ies6 is missing, and there is an increase in H2A.Z enrichment at these locations in ino 80 and ies 6 mutant strains. Moreover, the increase in ploidy associated with ies 6 mutant strains is greatly exacerbated by overexpression of H2A.Z and delayed by reduced H2A.Z expression. These findings point to pericentric chromatin as a key player in centromere function in budding yeast and further support the emerging model in which the chromatin organization and structure of point centromeres is more similar to that of regional centromeres than previously thought. 
Recently, nucleosome remodeling by RSC and ISW2 was found to play a role in regulating histone turnover at pericentric chromatin in budding yeast, and this was proposed to contribute to maintenance of kinetochore organization (Verdaasdonk et al. 2012). Our data suggest that INO80-dependent regulation of H2A.Z localization in pericentric chromatin may also contribute to this function. One possibility is that H2A.Z in pericentric chromatin actively helps orient or promote kinetochore attachments, which would make absence less of a problem than misincorporation, since its aberrant localization may drive incorrect attachments.

The function of INO80 in preventing polyploidy and defects in chromosome segregation may be conserved in other organisms. One study found that depletion of the Ino80 catalytic subunit in human cells results in increased aneuploidy and chromosome structural abnormalities (Hur et al. 2010). The YY1 protein, which is not conserved in yeast, is associated with INO80 in higher eukaryotes. Interestingly, loss of YY1 also results in polyploidy and increased chromosome structural aberrations in mouse cells (Wu et al. 2007). Consequently, the function of INO80 in facilitating proper chromosome segregation, in addition to its previously characterized roles in DNA repair and replication, could have profound implications in human disease and prevention of cancer.

\section{Materials and methods}

Yeast strains and plasmids

Yeast strains used in this study are listed in Table 2. Plasmid pJD640 contains the wild-type IES6 ORF plus promoter and terminator sequences cloned into the NotI site of pRS416 (Stratagene). The H2A.Z overexpression construct (in pBG1805) was from Open Biosystems.

\section{Flow cytometry analysis}

Samples analyzed in Figures $2 \mathrm{C}$ and $3 \mathrm{C}$ were analyzed as described in Bellay et al. (2011). For all other samples, cultures were grown in YPAD, -URA/glu, or -URA/gal medium to mid$\log$, and samples were taken and fixed in ethanol. Cells were harvested and incubated in $1 \mathrm{mg} / \mathrm{mL}$ RNaseA for $4 \mathrm{~h}$ at $37^{\circ} \mathrm{C}$, followed by incubation for $1 \mathrm{~h}$ at $50^{\circ} \mathrm{C}$ in $2 \mathrm{mg} / \mathrm{mL}$ proteinase $\mathrm{K}$ solution. Yeast cells were then collected by centrifugation and resuspended FACS buffer $(200 \mathrm{mM}$ Tris- $\mathrm{HCl}$ at $\mathrm{pH} 7.5,200 \mathrm{mM}$ $\mathrm{NaCl}, 78 \mathrm{mM} \mathrm{MgCl} 2$ ). Cells were added to $50 \mu \mathrm{g} / \mathrm{mL}$ propidium iodide in $50 \mathrm{mM}$ Tris- $\mathrm{HCl}$ (pH 7.5), sonicated, and analyzed using a FACSCalibur.

\section{CGH on microarray}

CGH was performed as described previously (Dion and Brown 2009). Genomic DNA from each strain was collected after growth for 40 or 80 generations post-sporulation for strains derived from IES6/ies6 or 20 or 40 generations post-sporulation for strains derived from INO80/ino80 heterozygous diploids. Experiment signal intensities from the microarrays were compared with wild-type controls using Tiling Analysis software (Affymetrix) using quantile normalization, perfect match probes only, a bandwidth of 60 , a maximum gap of 80 , and a minimum run of 40 . The CGH profiles were generated with IGB 6.3 (Affymetrix).

\section{Plasmid retention assay}

BY4741, ino80, or ies6 strains were transformed with either the CEN/ARS plasmid pRS415 or the $2-\mu \mathrm{m}$ plasmid pRS426 and were grown on medium lacking leucine or uracil, respectively. Multiple independent transformants from each strain were used to inoculate liquid synthetic complete (SC) medium and grown overnight. Overnight cultures were subcultured to an $\mathrm{OD}_{600}$ corresponding to 0.25 and grown for a further $4 \mathrm{~h}$ at $30^{\circ} \mathrm{C}$ to mid-log phase in nonselective medium before being plated onto both SC medium and medium lacking leucine or uracil. Colonies were counted after 3-5 d of incubation at $30^{\circ} \mathrm{C}$, and the percent plasmid retention was calculated as survival on selective medium (total viable cells) relative to that on nonselective medium (viable cells retaining plasmid). A minimum of three independent colonies were tested for each strain, and results are expressed as the mean percentage plasmid retention $\pm 1 \mathrm{SD}$.

\section{Spot tests}

Mid-log-phase cultures were diluted to a density corresponding to an absorbance of 0.2 at $600 \mathrm{~nm}$, and fivefold serial dilutions were plated onto medium containing the indicated concentration of $\mathrm{HU}$ or benomyl. Plates were incubated for 3$4 \mathrm{~d}$ at $30^{\circ} \mathrm{C}$.

\section{Indirect end label analysis}

Chromatin was digested with MNase in uncross-linked, Nonidet P40-permeabilized yeast cells using the general method described in Kent et al. (1993). Cells were grown to early log phase $\left(\sim 1.5 \times 10^{7}\right.$ cell per milliliter $)$ in YPD medium. All chromatin samples contained DNA equivalent to $2.0 \times 10^{8}$ nucleated cells and were digested with 75 and $150 \mathrm{U} / \mathrm{mL}$ MNase (USB) for 2.5 min at $37^{\circ} \mathrm{C}$. Equivalent amounts of purified genomic DNA were digested with $10 \mathrm{U} / \mathrm{mL}$ MNase for 30-50 sec at room temperature to provide deproteinized DNA controls. MNasetreated samples were digested to completion with the appropriate restriction enzymes and analyzed by indirect end labeling: DNAs were separated on $1.5 \%$ agarose gels together with Norgen Mid-Ranger size markers and Southern blotted to nylon membranes (MSI/Osmonics). Probes were gel-purified DNA fragments amplified by PCR from yeast genomic DNA and radiolabeled by random priming (Stratagene). The CEN4 probe spanned positions +637 bp (HindIII site) and $+1179 \mathrm{bp}$ of the RMD1 ORF. The CEN12 probe spanned positions $+1832 \mathrm{bp}$ (EcoRV site) and +2232 bp of the YLR001C ORF. Hybridizations and washes were carried out in aqueous buffer at $64^{\circ} \mathrm{C}$ as described (Kent et al. 1994).

\section{ChIP of H2A.Z at centromeres}

The indicated strains were grown in YPAD before immunoprecipitation with anti-histone H2A.Z (Millipore, 07-718). ChIP assays were performed essentially as in Strahl-Bolsinger et al. (1997). Immunoprecipitated and input DNA was quantified by quantitative PCR. Enrichment was analyzed at regions flanking both sides of CEN4 and CEN12 (primer sequences available on request). ChIP signal was normalized to amplification from input DNA and expressed relative to wild type. Data shown are the mean enrichment \pm SD.

\section{Western blotting}

Wild-type, ies6, ies6hda1, hda1, and ino80 cultures were lysed by glass bead disruption, and proteins were precipitated with 
Table 2. Yeast strains used in this study

\begin{tabular}{|c|c|c|}
\hline Strain name & Genotype & Source \\
\hline BY4741 & 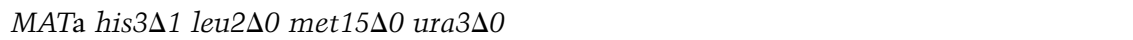 & Euroscarf \\
\hline BY4742 & $M A T \alpha$ his $3 \Delta 1$ leu2 $\Delta O$ lys $2 \Delta O$ ura3 $\Delta O$ & Euroscarf \\
\hline BY4743 & 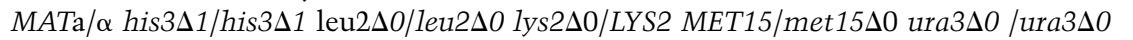 & Euroscarf \\
\hline ies1 & 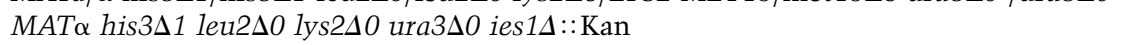 & Open Biosystems \\
\hline ies2 & 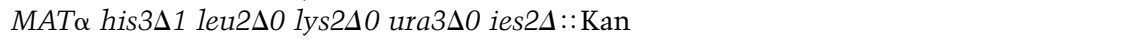 & Open Biosystems \\
\hline ies3 & 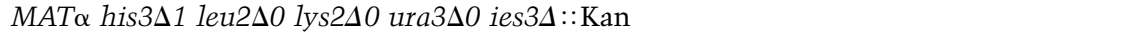 & Open Biosystems \\
\hline ies4 & 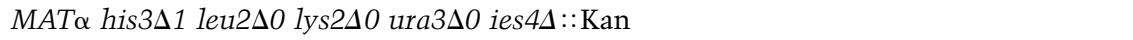 & Open Biosystems \\
\hline ies5 & 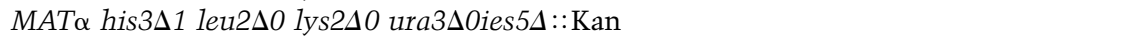 & Open Biosystems \\
\hline ies6 & 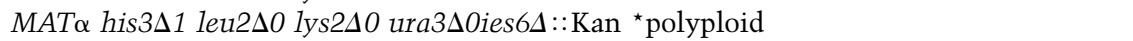 & Open Biosystems \\
\hline ies6 & 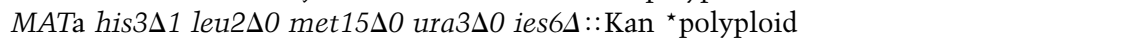 & Euroscarf \\
\hline $\operatorname{arp} 8$ & 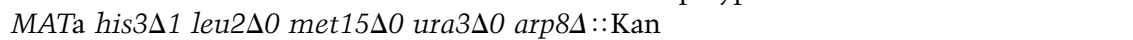 & Euroscarf \\
\hline ino80 & 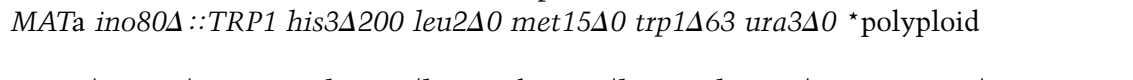 & $\begin{array}{l}\text { Gift from S. Shen and } \\
\text { C. Wu (Shen et al. 2000) }\end{array}$ \\
\hline JDY853 & 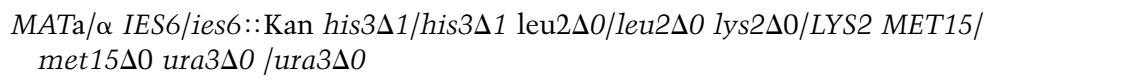 & This study \\
\hline INO80/ino80 & 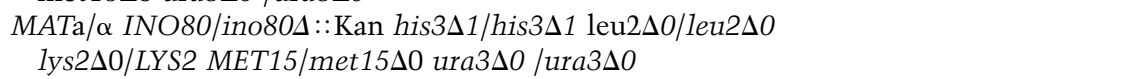 & Research Genetics \\
\hline JDY939 & 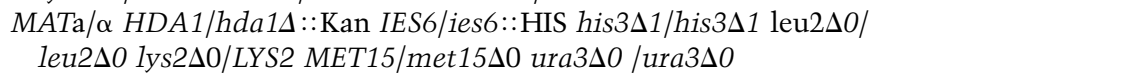 & This study \\
\hline JDY941 & hda1s::KanMX (JDY941-944 were spores derived from a single tetrad from JDY939) & This study \\
\hline JDY942 & Wild-type haploid (JDY941-944 were spores derived from a single tetrad from JDY939) & This study \\
\hline JDY943 & $\begin{array}{l}\text { ies6 } 6 \text { ::HisMX (JDY941-944 were spores derived from a single tetrad from JDY939) } \\
{ }^{*} \text { polyploid }\end{array}$ & This study \\
\hline JDY944 & $\begin{array}{l}\text { hda1 }:: \text { KanMX ies6::HisMX (JDY941-944 were spores derived from a single tetrad } \\
\text { from JDY939) *polyploid }\end{array}$ & This study \\
\hline ies $6 \Delta-A$ & ies64::KanMX (spore derived from JDY853) *polyploid & This study \\
\hline ies $6 \Delta-B$ & ies64::KanMX (spore derived from JDY853) * polyploid & This study \\
\hline JDY891 & ies64::KanMX (spore derived from JDY853) * polyploid & This study \\
\hline JDY895 & ies64::KanMX (spore derived from JDY853) * polyploid & This study \\
\hline ino80 $\Delta-A$ & ino804::KanMX (spore derived from INO80/ino80) * polyploid & This study \\
\hline ino80 $\Delta-B$ & ino804::KanMX (spore derived from INO80/ino80) *polyploid & This study \\
\hline JDY973 & Tetraploid created by mating JDY891 $\times$ JDY895 & This study \\
\hline JDY979 & 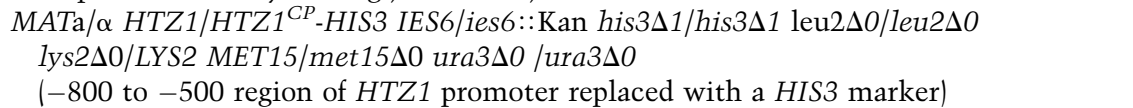 & This study \\
\hline JDY990 & $\begin{array}{l}\text { ies6 } 6 \text { ::KanMX (JDY990-993 were spores derived from a single tetrad from JDY979) } \\
{ }^{*} \text { polyploid }\end{array}$ & This study \\
\hline JDY991 & Wild-type haploid (JDY990-993 were spores derived from a single tetrad from JDY979) & This study \\
\hline JDY992 & $H T Z 1^{C P}$-HisMX (JDY990-993 were spores derived from a single tetrad from JDY979) & This study \\
\hline JDY993 & $\begin{array}{l}\text { ies6 } 6 \text { ::KanMX HTZ1 }{ }^{C P} \text {-HisMX (JDY990-993 were spores derived from a single tetrad } \\
\text { from JDY979) * polyploid }\end{array}$ & This study \\
\hline TSY85 & 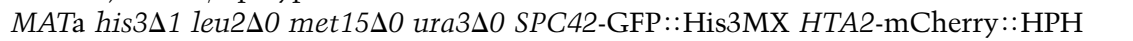 & This study \\
\hline TSY86 & 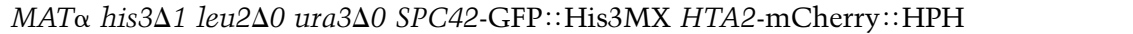 & This study \\
\hline TSY87 & 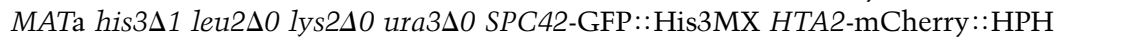 & This study \\
\hline TSY88 & 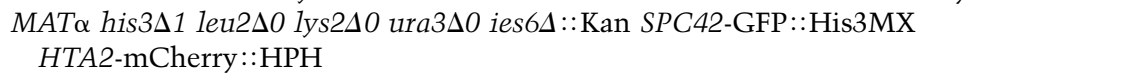 & This study \\
\hline TSY89 & 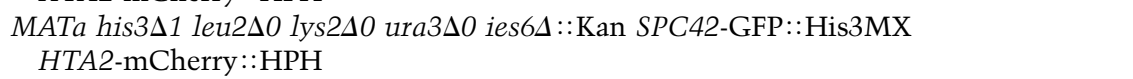 & This study \\
\hline TSY90 & 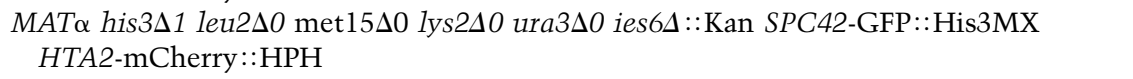 & This study \\
\hline
\end{tabular}

$20 \%$ TCA before resuspension in SDS loading buffer. Samples were analyzed by Western blotting using anti-H2A.Z (Millipore, 07-718) or anti-acetyl-Htzl (Lys14) (Millipore, 07-719) and an antibody raised against $\mathrm{H} 2 \mathrm{~A}$.

\section{Acknowledgments}

We are grateful to C. Wu and S. Shen for the ino80 yeast strain, to Ben Wardleworth for initial observations with IES6, and to Fred Winston, Manolis Papamichos-Chronakis, Craig Peterson, Kerry Bloom, and members of the Downs laboratory for helpful discussions. A.L.C. and J.A.D. are supported by Cancer Research UK CEA C7905/A10269. N.A.K. is supported by BBSRC grant BB/F012810/1. G.W.B. is supported by Canadian Institutes of Health Research grant MOP-84292, and T.L.S. is supported by a Natural Sciences and Engineering Council of Canada PostGraduate Scholarship.

\section{References}

Albert I, Mavrich TN, Tomsho LP, Qi J, Zanton SI, Schuster SC, Pugh BF. 2007. Translational and rotational settings of 
H2A.Z nucleosomes across the Saccharomyces cerevisiae genome. Nature 446: 572-576.

Andalis AA, Storchova Z, Styles C, Galitski T, Pellman D, Fink GR. 2004. Defects arising from whole-genome duplications in Saccharomyces cerevisiae. Genetics 167: 1109-1121.

Bao Y, Shen X. 2007. INO80 subfamily of chromatin remodeling complexes. Mutat Res 618: 18-29.

Bellay J, Atluri G, Sing TL, Toufighi K, Costanzo M, Ribeiro PS, Pandey G, Baller J, VanderSluis B, Michaut M, et al. 2011. Putting genetic interactions in context through a global modular decomposition. Genome Res 21: 1375-1387.

Boyarchuk E, Montes de Oca R, Almouzni G. 2011. Cell cycle dynamics of histone variants at the centromere, a model for chromosomal landmarks. Curr Opin Cell Biol 23: 266-276.

Carr AM, Dorrington SM, Hindley J, Phear GA, Aves SI, Nurse P. 1994. Analysis of a histone H2A variant from fission yeast: Evidence for a role in chromosome stability. Mol Gen Genet 245: 628-635.

Chen L, Cai Y, Jin J, Florens L, Swanson SK, Washburn MP, Conaway JW, Conaway RC. 2011. Subunit organization of the human INO80 chromatin remodeling complex: An evolutionarily conserved core complex catalyzes ATPdependent nucleosome remodeling. J Biol Chem 286: 11283 11289.

Collins SR, Miller KM, Maas NL, Roguev A, Fillingham J, Chu CS, Schuldiner M, Gebbia M, Recht J, Shales M, et al. 2007. Functional dissection of protein complexes involved in yeast chromosome biology using a genetic interaction map. $\mathrm{Na}$ ture 446: 806-810.

Conaway RC, Conaway JW. 2009. The INO80 chromatin remodeling complex in transcription, replication and repair. Trends Biochem Sci 34: 71-77.

Dion B, Brown GW. 2009. Comparative genome hybridization on tiling microarrays to detect aneuploidies in yeast. Methods Mol Biol 548: 1-18.

Falbo KB, Alabert C, Katou Y, Wu S, Han J, Wehr T, Xiao J, He X, Zhang Z, Shi Y, et al. 2009. Involvement of a chromatin remodeling complex in damage tolerance during DNA replication. Nat Struct Mol Biol 16: 1167-1172.

Foijer F. 2010. CINister thoughts. Biochem Soc Trans 38: 17151721.

Greaves IK, Rangasamy D, Ridgway P, Tremethick DJ. 2007. H2A.Z contributes to the unique 3D structure of the centromere. Proc Natl Acad Sci 104: 525-530.

Hawthorne DC. 1963. A deletion in yeast and its bearing on the structure of the mating type locus. Genetics 48: 17271729.

Hou H, Wang Y, Kallgren SP, Thompson J, Yates JR III, Jia S. 2010. Histone variant H2A.Z regulates centromere silencing and chromosome segregation in fission yeast. I Biol Chem 285: 1909-1918.

Hur SK, Park EJ, Han JE, Kim YA, Kim JD, Kang D, Kwon J. 2010. Roles of human INO80 chromatin remodeling enzyme in DNA replication and chromosome segregation suppress genome instability. Cell Mol Life Sci 67: 2283-2296.

Kawashima S, Nakabayashi Y, Matsubara K, Sano N, Enomoto T, Tanaka K, Seki M, Horikoshi M. 2011. Global analysis of core histones reveals nucleosomal surfaces required for chromosome bi-orientation. EMBO J 30: 3353-3367.

Kent NA, Bird LE, Mellor J. 1993. Chromatin analysis in yeast using NP-40 permeabilised sphaeroplasts. Nucleic Acids Res 21: 4653-4654.

Kent NA, Tsang JS, Crowther DJ, Mellor J. 1994. Chromatin structure modulation in Saccharomyces cerevisiae by centromere and promoter factor 1. Mol Cell Biol 14: 52295241 .
Kim HS, Vanoosthuyse V, Fillingham J, Roguev A, Watt S, Kislinger T, Treyer A, Carpenter LR, Bennett CS, Emili A, et al. 2009. An acetylated form of histone H2A.Z regulates chromosome architecture in Schizosaccharomyces pombe. Nat Struct Mol Biol 16: 1286-1293.

Krogan NJ, Baetz K, Keogh MC, Datta N, Sawa C, Kwok TC, Thompson NJ, Davey MG, Pootoolal J, Hughes TR, et al. 2004. Regulation of chromosome stability by the histone $\mathrm{H} 2 \mathrm{~A}$ variant $\mathrm{Htz} 1$, the Swr1 chromatin remodeling complex, and the histone acetyltransferase NuA4. Proc Natl Acad Sci 101: 13513-13518.

Measday V, Baetz K, Guzzo J, Yuen K, Kwok T, Sheikh B, Ding H, Ueta R, Hoac T, Cheng B, et al. 2005. Systematic yeast synthetic lethal and synthetic dosage lethal screens identify genes required for chromosome segregation. Proc Natl Acad Sci 102: 13956-13961.

Papamichos-Chronakis M, Peterson CL. 2008. The Ino80 chromatin-remodeling enzyme regulates replisome function and stability. Nat Struct Mol Biol 15: 338-345.

Papamichos-Chronakis M, Watanabe S, Rando OI, Peterson CL. 2011. Global regulation of H2A.Z localization by the INO80 chromatin-remodeling enzyme is essential for genome integrity. Cell 144: 200-213.

Pinto I, Winston F. 2000. Histone H2A is required for normal centromere function in Saccharomyces cerevisiae. EMBO I 19: $1598-1612$.

Rangasamy D, Berven L, Ridgway P, Tremethick DJ. 2003. Pericentric heterochromatin becomes enriched with H2A.Z during early mammalian development. EMBO J 22: 1599-1607.

Ranjitkar P, Press MO, Yi X, Baker R, MacCoss MJ, Biggins S. 2010. An E3 ubiquitin ligase prevents ectopic localization of the centromeric histone $\mathrm{H} 3$ variant via the centromere targeting domain. Mol Cell 40: 455-464.

Shen X, Mizuguchi G, Hamich A, Wu C. 2000. A chromatin remodeling complex involved in transcription and DNA processing. Nature 406: $541-544$.

Shen X, Ranallo R, Choi E, Wu C. 2003. Involvement of actinrelated proteins in ATP-dependent chromatin remodeling. Mol Cell 12: 147-155.

Shimada K, Oma Y, Schleker T, Kugou K, Ohta K, Harata M, Gasser SM. 2008. Ino80 chromatin remodeling complex promotes recovery of stalled replication forks. Curr Biol 18: 566-575.

Sopko R, Huang D, Preston N, Chua G, Papp B, Kafadar K, Snyder M, Oliver SG, Cyert M, Hughes TR, et al. 2006. Mapping pathways and phenotypes by systematic gene overexpression. Mol Cell 21: 319-330.

Storchova Z, Breneman A, Cande J, Dunn J, Burbank K, O'Toole E, Pellman D. 2006. Genome-wide genetic analysis of polyploidy in yeast. Nature 443: 541-547.

Storchova Z, Becker JS, Talarek N, Kogelsberger S, Pellman D. 2011. Bub1, Sgo1, and Mps1 mediate a distinct pathway for chromosome biorientation in budding yeast. Mol Biol Cell 22: $1473-1485$

Strahl-Bolsinger S, Hecht A, Luo K, Grunstein M. 1997. SIR2 and SIR4 interactions differ in core and extended telomeric heterochromatin in yeast. Genes Dev 11: 83-93.

Torras-Llort M, Moreno-Moreno O, Azorin F. 2009. Focus on the centre: The role of chromatin on the regulation of centromere identity and function. EMBO J 28: 2337-2348.

Tsukuda T, Fleming AB, Nickoloff JA, Osley MA. 2005. Chromatin remodelling at a DNA double-strand break site in Saccharomyces cerevisiae. Nature 438: 379-383.

Verdaasdonk JS, Bloom K. 2011. Centromeres: Unique chromatin structures that drive chromosome segregation. Nat ReV Mol Cell Biol 12: 320-332. 
Verdaasdonk JS, Gardner R, Stephens AD, Yeh E, Bloom K. 2012. Tension dependent nucleosome remodeling at the pericentromere in yeast. Mol Biol Cell 23: 2560-2570.

Wu S, Shi Y, Mulligan P, Gay F, Landry J, Liu H, Lu J, Qi HH, Wang W, Nickoloff JA, et al. 2007. A YY1-INO80 complex regulates genomic stability through homologous recombination-based repair. Nat Struct Mol Biol 14: 1165-1172.

Yu Y, Srinivasan M, Nakanishi S, Leatherwood J, Shilatifard A, Sternglanz R. 2011. A conserved patch near the C terminus of histone $\mathrm{H} 4$ is required for genome stability in budding yeast. Mol Cell Biol 31: 2311-2325. 


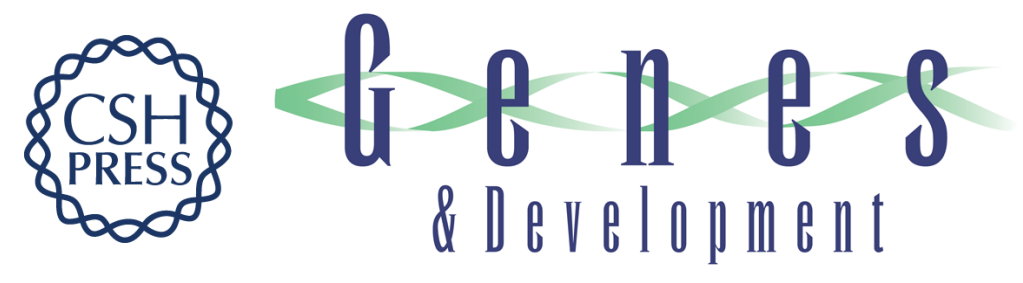

\section{The INO80 chromatin remodeling complex prevents polyploidy and maintains normal chromatin structure at centromeres}

Anna L. Chambers, Georgina Ormerod, Samuel C. Durley, et al.

Genes Dev. 2012, 26:

Access the most recent version at doi:10.1101/gad.199976.112

Supplemental http://genesdev.cshlp.org/content/suppl/2012/11/28/26.23.2590.DC1
Material

References This article cites 40 articles, 13 of which can be accessed free at:

http://genesdev.cshlp.org/content/26/23/2590.full.html\#ref-list-1

License

Email Alerting

Receive free email alerts when new articles cite this article - sign up in the box at the top

Service

right corner of the article or click here.

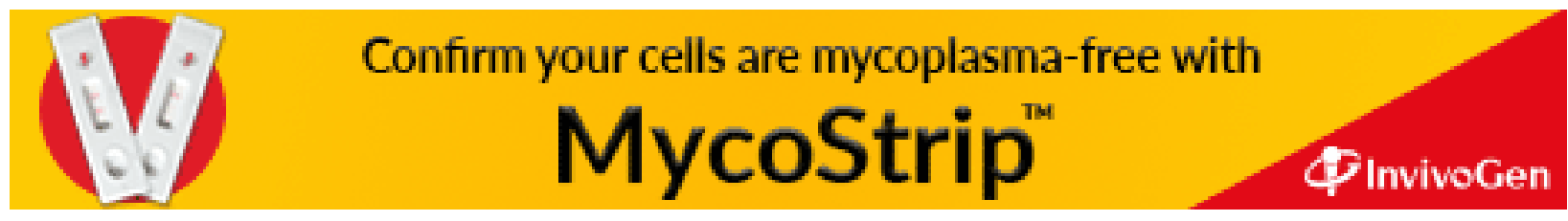

